\title{
Perthen, Andrea: Korruption kritisieren. Die Genese politischer Korruptionsskandale in der frühen Bundesrepublik Deutschland.
}

\author{
Köln: Herbert von Halem 2021. 380 Seiten. Preis: $€ 36,00$
}

\section{Konrad Dussel}

Angenommen: 22. Dezember 2021 / Online publiziert: 11. Januar 2022

(C) Der/die Autor(en) 2022

Der bis heute bekannteste politische Skandal der 1960er-Jahre und vielleicht der bundesrepublikanischen Geschichte schlechthin ist die Spiegel-Affäre des Jahres 1962. Doch vom Kontext ist wenig in Erinnerung geblieben. Eigentlich bildet sie nur den dramatischen Höhepunkt einer ganzen Reihe von mehr oder minder großen Skandalen, in der zwei Protagonisten die Hauptrollen spielten: der zeitweilige Verteidigungsminister Franz-Josef Strauß und Spiegel-Herausgeber Rudolf Augstein. Neben der so genannten ,Fibag- " und der ,Onkel-Aloys-Affäre" war es vor allem der HS-30-Skandal, der sogar zweimal - Ende der 1950er und Ende der 1960er-Jahre den politisch interessierten Teil der Republik erschütterte. Wie beim StarfighterSkandal ging es auch bei ihm um dubiose Beschaffungsmaßnahmen für die neu aufzubauende Bundeswehr, in diesem Fall um einen Schützenpanzer, von dem zunächst mehr als 10.000 Stück bei einer Schweizer Firma geordert wurden, ohne dass diese einschlägige Erfahrungen oder auch nur einen Prototypen hätte vorweisen können. Der Verdacht lag da nahe, dass im Vorfeld umfangreiche Gelder geflossen wären zum einen an die CDU als Regierungspartei, zum anderen aber auch ganz konkret an einzelne ihrer Mitglieder, vor allem an den 1957 unter mysteriösen Umständen verstorbenen früheren Staatssekretär Otto Lenz.

So betrachtet böte das Thema der 2019 an der Technischen Universität Darmstadt angenommenen Dissertation hinreichend Stoff für eine umfassende Analyse und spannende Darstellung. Doch das Buch von Andrea Perthen erfüllt beide Hoffnungen höchstens ansatzweise. Perthen gelingt es nicht, bei der Behandlung ihres komplexen Stoffes klare Prioritäten zu setzen. Zu häufig verliert sie sich in der vergleichsweise ausführlichen Darstellung von Nebensächlichkeiten - wie etwa die Entwicklung des deutschen Journalismus überhaupt einschließlich von Rundfunk und Presse oder

Apl. Prof. Dr. Konrad Dussel ( $₫)$

Historisches Institut, L 7, 7, Universität Mannheim, 68161 Mannheim, Deutschland

E-Mail: Konrad.Dussel@t-online.de 
die Charakterisierung einzelner Informanten bei den geschilderten Skandalen -, zu wenig diskutiert sie dagegen die Motive der Protagonisten. Sicherlich, schon früh stellt Perthen das ,,besondere “Verhältnis“ von Augstein zu Strauß heraus und seine Versuche, „Strauß massiv zu bekämpfen und politisch unmöglich zu machen“ (S. 62). Durch das völlige Ausblenden der Spiegel-Affäre gerät aber dessen Antwort nicht so recht in den Blick, auf die wiederum Augsteins Replik in Form der erneuten Behandlung des HS-30-Problems Ende der 1960er-Jahre folgte.

Nun wäre es verkürzt, nur von der Auseinandersetzung zwischen Augstein und Strauß zu sprechen. Wichtige Rollen spielten auch Peter Miska, Bernt Engelmann und Gert von Paczensky. Die besondere Rolle Paczenskys im kritischen Journalismus der 1960er-Jahre wird von Perthen zwar angedeutet, aber nicht angemessen vertieft. Und weil sie auch nicht wirklich klären mag, was damals bei den Skandalen alles geschah, bleibt ein gewisses Gefühl des Unbefriedigtseins. Vielleicht war tatsächlich doch alles nicht so eindeutig, wie es die ,Skandalisierer“ darstellten, und vielleicht hatte der spätere Bundeskanzler Schmidt recht, als er 1966 einem Interessierten im Rückblick auf die erste Runde des HS-30-Skandals schrieb, „bedauerlicherweise sei der ,Beweiswert" der Veröffentlichung nicht gar so ,erdrückend" wie von diesen dargestellt“ (S. 323). Perthen trägt zwar durchaus eine Menge Detailinformationen zusammen, eine kohärente Analyse ist ihr jedoch nicht gelungen.

Sicherlich wäre eine geschicktere Dramaturgie der Darstellung auch bei einer Dissertation erfreulich. Das bildet jedoch kein zentrales Kriterium. Ärgerlicher ist da schon das seltsame Belegsystem, das sowohl den amerikanischen als auch den europäischen Stil verwendet und dabei auch noch eigene Modifikationen einbringt. In den 600 Fußnoten findet man primär in Historiker-Tradition Quellenbelege, die Literaturbelege im amerikanischen Stil dagegen im Text. Es sei denn, es handelt sich um Zeitungs- oder Zeitschriftenquellen; die stehen auch im Text. Da sich auch immer wieder wichtige ergänzende Informationen in den Fußnoten finden, kommt man nicht umhin, auch diese gründlich zu lesen.

Funding Open Access funding enabled and organized by Projekt DEAL.

Open Access Dieser Artikel wird unter der Creative Commons Namensnennung 4.0 International Lizenz veröffentlicht, welche die Nutzung, Vervielfältigung, Bearbeitung, Verbreitung und Wiedergabe in jeglichem Medium und Format erlaubt, sofern Sie den/die ursprünglichen Autor(en) und die Quelle ordnungsgemäß nennen, einen Link zur Creative Commons Lizenz beifügen und angeben, ob Änderungen vorgenommen wurden.

Die in diesem Artikel enthaltenen Bilder und sonstiges Drittmaterial unterliegen ebenfalls der genannten Creative Commons Lizenz, sofern sich aus der Abbildungslegende nichts anderes ergibt. Sofern das betreffende Material nicht unter der genannten Creative Commons Lizenz steht und die betreffende Handlung nicht nach gesetzlichen Vorschriften erlaubt ist, ist für die oben aufgeführten Weiterverwendungen des Materials die Einwilligung des jeweiligen Rechteinhabers einzuholen.

Weitere Details zur Lizenz entnehmen Sie bitte der Lizenzinformation auf http://creativecommons.org/ licenses/by/4.0/deed.de.

Apl. Prof. Dr. Konrad Dussel ist apl. Professor für Neuere Geschichte an der Universität Mannheim. 